\title{
Temporal Resolution Ability in Students with Dyslexia and Reading and Writing Disorders
}

\author{
Juliana Chaubet ${ }^{1} \quad$ Liliane Pereira $^{2} \quad$ Ana Paula Perez ${ }^{3}$ \\ 1 Specialist at the Program in Human Communication Disorders - \\ Speech and Hearing Department - Universidade de São Paulo, Brazil \\ ${ }^{2}$ Associate Professor - Program in Human Communication Disorders - \\ Speech and Hearing Department - Universidade de São Paulo, Brazil \\ $3 \mathrm{PhD}$ at Program in Human Communication Disorders - Speech and \\ Hearing Department - Universidade de São Paulo, Brazil. Assistant \\ Professor of the Department of Speech Language Patology - \\ Universidade Federal Fluminense, Brazil
}

Int Arch Otorhinolaryngol 2014;18:146-149.

\begin{abstract}
Address for correspondence Ana Paula Perez, Specific Graduation in Speech Language Patology, Universidade Federal Fluminense, Nova Friburgo, Rio de Janeiro, Brazil (e-mail: popiperez@ig.com.br).
\end{abstract}

\begin{abstract}
Keywords

- auditory perception

- hearing tests

- dyslexia
\end{abstract}

Introduction The Gaps-in-Noise (GIN) test assesses the hearing ability of temporal resolution. The development of this ability can be considered essential for learning how to read. Objective Identify temporal resolution in individuals diagnosed with reading and writing disorders compared with subjects with dyslexia.

Methods A sample of 26 subjects of both genders, age 10 to 15 years, included 11 diagnosed with dyslexia and 15 diagnosed with reading and writing disorders. Subjects did not display otologic, neurologic, and/or cognitive diseases. A control group of 30 normal-hearing subjects was formed to compare thresholds and percentages obtained from the GIN test. The responses were obtained considering two measures of analysis: the threshold gap and the percentage of correct gap.

Results The threshold was lower in the GIN for the typical group than for the other groups. There was no difference between groups with dyslexia and with reading and writing disorders. The GIN results of the typical group revealed a higher percentage of correct answer than in the other groups. No difference was obtained between the groups with dyslexia and with reading and writing disorders.

Conclusion The GIN test identified a difficulty in auditory ability of temporal resolution in individuals with reading and writing disorders and in individuals with dyslexia in a similar way.

\section{Introduction}

The ability of temporal resolution is fundamental for comprehension of human speech and is a prerequisite for linguistic abilities. ${ }^{1,2}$ This ability acts in the auditory perception of verbal and nonverbal sounds; in the perception of music, rhythms, and periodicity; and in the discrimination of steps, duration, and phonemes. ${ }^{3}$

Alteration of the auditory perception may bring about problems in speech and language development as well as in learning and socialization of children, adults, and the elderly.
The decoding of the spoken message involves the analysis of many signal components including the acoustical, phonetic, phonological, lexical, suprasegmental, syntactical, and semantic components. ${ }^{4,5}$ For this decoding to occur, the acoustical cues of frequency, intensity, and timing must be processed in a precise manner by the auditory system. ${ }^{6,7}$

Phonemes, the isolated sounds that make up the syllables, represent these rapid sound stimuli in speech. Hence, the individual with altered perception would have difficulty perceiving that words can be decomposed in those smaller isolated sounds. This ability, called phonological conscience, is received

September 6, 2013

accepted

September 17, 2013
DOI http://dx.doi.org/

10.1055/s-0033-1363465. ISSN 1809-9777.
Copyright $\odot 2014$ by Thieme Publicações License terms Ltda, Rio de Janeiro, Brazil

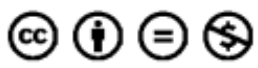


essential for one to master the alphabetical system in which the phonemes are mapped as letters (graphemes). Therefore, the learning of this ability can be considered essential when learning how to $\operatorname{read}^{8}$ and at the same time is the main manifestation of the reading and writing disorders.

According to the World Health Organization, ${ }^{9}$ dyslexia is defined as a disorder that manifests itself by specific difficulties in learning how to read and write that are not attributable to any other kind of deficit related to intelligence, motivation, learning opportunity, or sensorial acuity. Reading and writing disorder is described as a manifestation that features the difficulty in acquiring and/or developing written language by children and presents both deficits in phonological decoding and oral and/or written language comprehension. ${ }^{10}$

Keeping this relation in mind, subjects with phonological deviation and/or reading and writing difficulties may present alterations in temporal auditory processing and may need more time to detect gaps between auditory stimuli.

The goal of this study was to verify and compare the temporal resolution ability in individuals diagnosed with a reading and writing disorder to those with dyslexia, which may help in speech and language rehabilitation.

\section{Methods}

This study was approved by the Universidade Federal de São Paulo Ethics in Research Committee (UNIFESP/EPM), process no. 1726/09. All the selected subjects were volunteers and signed a free and willing consent form.

Participating subjects were divided in two groups: 11 with the diagnosis of dyslexia (GD) and 15 with the diagnosis of reading and writing disorder (GSD). Subjects in the GSD group were receiving speech and language treatment at the Nucleus for Teaching, Assistance and Research of Reading and Writing Disorders - NEAPEL - UNIFESP. For patients with a diagnosis of dyslexia, the Brazilian Association of Dyslexia and Infant Interdisciplinary Nucleus of Neuropsychological Treatment were contacted.

To form this study sample, volunteers had to be between the ages of 10 and 15, of either gender, without any history of evidence of psychiatric or neurologic impairment or of hearing loss.

To constitute the control group, the work of Perez and Pereira ${ }^{11}$ was used to establish normalcy reference criteria for youngsters of 11 and 12 years of age of both genders. The threshold measure considered as the normal value followed the criteria of a positive response for four of six presentations: a 5.0-millisecond threshold for the right ear and 5.11 milliseconds for the left ear, and a total $71.99 \%$ correct response for the right ear and $71.41 \%$ for the left. A stratified lottery based on the studied age groups was performed and 21 subjects were selected.

All subjects completed a basic hearing assessment, including collection of a case history, tonal threshold audiometry, speech audiometry, and tympanometry.

The procedure used to measure the behavior of temporal resolution was the Gaps-in-Noise (GIN) test proposed by Musiek et $\mathrm{al}^{12}$ with a total of 60 silence intervals or gaps inserted in a noise segment, with a time variation of silence between 2 and 20 milliseconds. Two kinds of responses were analyzed: the temporal acuity threshold, which is the least value in milliseconds in which the subject perceived the silence interval, and the total number of correctly identified gaps, in percentage values.

The GIN test, presented from a CD, was applied using an Interacoustic MA-41 audiometer attached to a Sony CD player (Interacoustics AS Drejervaenget, Assens0 Denmark) in an acoustic cabin at a 50-dB SL (sensation level) intensity (according to the mean auditory thresholds for 500, 1,000, and $2,000 \mathrm{~Hz}$ ). All the results were recorded in specific registration sheets. The $\mathrm{CD}$ is composed of a training track and four test tracks. Each test track is composed of diverse stimuli of 6 seconds of white noise with 5-second intervals between the stimuli.

The training track was applied before the beginning of the test to ensure that the subject clearly understood what needed to be done. The test was applied to one ear at a time. In the right ear, test track 2 was used, and in the left ear, test track 3. The instructions to the subjects were: "You will hear a noise and within this noise there will be spaces or gaps in which the noise will be absent. The gaps will vary in size and you will have to listen carefully, because some of them will be extremely small. Occasionally there will be no gaps. You must raise your hand every time you hear a gap."

The threshold considered was the perception of four of six presentations of the same gap, that is, $66.66 \%$ of identification of a specific silence interval in milliseconds according to the criteria of Musiek et al. ${ }^{12}$

To assess the answers in this study, in the three groups (control, diagnosis of reading and writing disorder, diagnosis of dyslexia), the nonparametric test of Kruskal-Wallis was used, followed by a procedure of multiple nonparametric comparisons. A nonparametric test was applied because it was not possible to find a transformation that could stabilize the variance in the responses of the three groups. The criteria adopted to determine the significance was the level of 0.05 with the construction of confidence intervals of $95 \%$.

\section{Results}

- Table 1 shows the descriptive statistics for the responses obtained by the three groups: the group with dyslexia, the group with reading and writing disorders, and the control group. The GIN threshold was not the same for all groups for both the right $(p<0.001)$ and the left ears $(p=0.0015)$; it was significantly lower for the control group than for the other groups ( $p<0.05$ ). There was no difference between the group with dyslexia and the group with reading and writing disorders $(p>0.05)$.

The percentage of correct responses for the GIN was not the same for the three groups, for both the right $(p<0.001)$ and left ears $(p<0.001)$. The GIN percentage was significantly higher for the control group than for the other groups $(p<0.05)$. There was no difference between the group with dyslexia and the group with reading and writing disorders $(p>0.05)$. 
Table 1 Descriptive measures of the gap thresholds (in milliseconds) and percentage of correct gap identification for each group for the right and left ears

\begin{tabular}{|l|l|l|l|l|l|l|l|l|l|}
\hline Group & Variable & Mean & SD & $\mathbf{0} \%$ & $\mathbf{2 5 \%}$ & $\mathbf{5 0 \%}$ & $\mathbf{7 5 \%}$ & $\mathbf{1 0 0} \%$ & No. of subjects \\
\hline \multirow{4}{*}{ Control } & GIN T RE & 4.6 & 0.57 & 4 & 4 & 5 & 5 & 6 & 21 \\
\cline { 2 - 10 } & GIN T LE & 5.0 & 0.89 & 4 & 5 & 5 & 5 & 8 & 21 \\
\cline { 2 - 10 } & \% GIN RE & 73.33 & 5.29 & 60.00 & 71.67 & 71.67 & 75.00 & 85.00 & 21 \\
\cline { 2 - 10 } & $\%$ GIN LE & 72.65 & 6.01 & 58.33 & 70.00 & 73.33 & 76.67 & 81.67 & 21 \\
\hline GSD & GIN T RE & 7.8 & 3.76 & 4 & 5.5 & 6 & 10 & 15 & 15 \\
\cline { 2 - 10 } & GIN T LE & 7.4 & 2.72 & 4 & 4.5 & 8 & 10 & 12 & 15 \\
\cline { 2 - 10 } & $\%$ GIN RE & 57.44 & 18.22 & 25.00 & 42.49 & 56.66 & 69.99 & 88.33 & 15 \\
\cline { 2 - 10 } & $\%$ GIN LE & 60.67 & 16.39 & 31.66 & 47.49 & 61.66 & 75.00 & 85.00 & 15 \\
\hline GD & GIN T RE & 7.1 & 1.16 & 5 & 6 & 8 & 8 & 8 & 11 \\
\cline { 2 - 9 } & GIN T LE & 7.2 & 1.27 & 5 & 7 & 8 & 8 & 8 & 11 \\
\cline { 2 - 9 } & $\%$ GIN RE & 55.60 & 5.83 & 43.33 & 53.33 & 56.66 & 58.33 & 65.00 & 11 \\
\cline { 2 - 9 } & $\%$ GIN LE & 55.60 & 8.03 & 48.00 & 49.16 & 55.00 & 60.83 & 70.00 & 11 \\
\hline
\end{tabular}

Abbreviations: GD, group with dyslexia; GIN, Gaps-in-Noise test; GSD, group with specific reading and writing disorder; LE, left ear RE, right ear; SD, standard deviation; T, threshold.

\section{Discussion}

Analysis of the GIN test in our subjects with dyslexia and subjects with a reading and writing disorder reinforce studies that indicate that phonological alterations need a longer temporal processing, that is, need a longer interval to perceive the difference between sounds. Some researchers indicated that school-aged children with dyslexia may present temporal processing problems. ${ }^{13}$ The perceptual abilities related to speech, language, and reading are extremely dependent on the ability of temporal resolution, which may be assessed by the GIN test.

In the present study, school-aged children in the group with dyslexia and the group with reading and writing disorders presented a deficit in auditory temporal resolution compared with the control group. There was no statistically significant difference between the responses for the study group with dyslexia and the group with reading and writing disorders.

Some studies verified that the phonological deficit must be caused by an auditory processing deficit due to the fact that the altered auditory temporal processing may hinder the perception of subtle signals in speech resulting in the observed difficulties in phonological processing. ${ }^{13-16}$ Boscariol et al studied the Random Gap Detection Test (RGDT) and found results of 13 milliseconds for the control group and 32.39 milliseconds for the GD group. ${ }^{13}$ The RGDT and GIN tests assess the temporal resolution ability; however, they have diverse investigation natures, hence there were differences in threshold limits for the same sample between test protocols. $^{17}$

Simões and Schochat assessed 40 children aged 7 to 12 years of age, 20 with dyslexia and 20 in a group with auditory processing disorders. ${ }^{14}$ The tests involved the abili- ties of auditory closure, figure-ground for linguistic sounds, and temporal ordering. The subjects in the dyslexia group presented altered responses only for the test that assessed temporal processing.

These researches agree with other studies that associate reading and writing abilities to auditory temporal processing abilities. $^{15,18}$

Regarding the descriptive measures of the auditory responses for the GIN test, we observed a gap threshold for the right ear of 7.8 milliseconds for the group with reading and writing disorders and a gap of 7.1 milliseconds for the group with dyslexia. The left ear obtained the threshold of 7.4 milliseconds for the group with reading and writing disorders and 7.2 milliseconds for the group with dyslexia. The found percentage was $55.60 \%$ for both ears for the dyslexia group, and for the reading and writing disorders was $57.44 \%$ for the right ear and $60.77 \%$ for the left ear.

The literature points to gap thresholds less than 6 milliseconds in subjects from 7 to 46 years of age. This confirms that the subjects with dyslexia and with reading and writing disorders present worse results when compared with the control group.

Samelli and Schochat ${ }^{19}$ observed similar gap detection thresholds for both ears when studying 100 young adults age 18 to 31 . The general mean of the gap thresholds was 3.98 milliseconds and the mean percentage of correct gap detection was $78.89 \%$. However, the author considered a gap threshold to be $50 \%$ of presentations, that is, three correct responses of the six presented. A study in school-aged children found values of 4.7 milliseconds and $73.6 \%$ in children of 8,9 , and 10 years of age $e^{20}$ and 5.05 milliseconds and $71.70 \%$ in children age 11 and 12 years. ${ }^{11}$

The group with dyslexia and the group with the diagnosis of reading and writing disorders in our study had lower mean 
response values than those obtained for the control group and lower than that in the reviewed literature, indicating the presence of the inability of temporal resolution in individuals with reading/writing difficulties.

\section{Conclusion}

After analyzing the data obtained in this study, subjects in the group with dyslexia and in the group with reading and writing disorders presented worse responses in the GIN test than the control group. This result can indicate a correlation between the temporal processing abilities and the reading and writing abilities.

\section{References}

1 Leitner DS, Hammond GR, Springer CP, et al. Parameters affecting gap detection in the rat. Percept Psychophys 1993;54(3): 395-405

2 Eggermont JJ. Neural responses in primary auditory cortex mimic psychophysical, across-frequency-channel, gap-detection thresholds. J Neurophysiol 2000;84(3):1453-1463

3 Zaidan E, Garcia AP, Tedesco MLF, Baran JA. Performance of normal oung adults in two temporal resolution tests. Pro Fono 2008;20(1): 19-24

4 Nicholas JG, Geers AE. Effects of early auditory experience on the spoken language of deaf children at 3 years of age. Ear Hear 2006; 27(3):286-298

5 Trehub SE, Henderson JL. Temporal resolution in infancy and subsequent language development. J Speech Hear Res 1996; 39(6):1315-1320

6 Lubert N. Auditory perceptual impairments in children with specific language disorders: a review of the literature. J Speech Hear Disord 1981;46(1):1-9

7 Amaral MI, Colella Santos MF. Temporal resolution: performance of school-aged children in the GIN -Gaps-in-noise test. Braz J Otorhinolarngol 2010. Nov-Dec; 76(6):745-52
8 Temple E, Poldrack RA, Salidis J, et al. Disrupted neural responses to phonological and orthographic processing in dyslexic children: an fMRI study. Neuroreport 2001;12(2):299-307

9 World Health Organization. ICD-10: The International Classification of Diseases. Geneva, Switzerland: World Health Organization; 1993

10 Santos MTM, Navas ALP. Terapia da linguagem escrita. In: Distúrbios de leitura e escrita: teoria e prática. Barueri, Brazil: Manole; 2002:191-223

11 Perez AP, Pereira LD. The Gap in Noise test in 11 and 12 year-old children. Pro Fono 2010 Jan-Mar, 22(1):7-12

12 Musiek FE, Zaidan EP, Baran JA, Shinn JB, Jirsa RE. Assessing temporal processes in adults with LD: the GIN test. In: American Academy of Audiology Convention. March-April. 2004. Salt Lake City. Annals Salt Lake City. UT: April 2, 2004:203pp

13 Boscariol M, Guimarães CA, Hage SRV, Cendes F, Guerreiro MM. Temporal auditory processing: correlation with developmental dyslexia and cortical malformation. Pro Fono 2010;22(4):537-42

14 Simões MB, Schochat E. (Central) auditory processing disorders in individuals with and without dyslexia. Pro Fono 2010;22(4): $521-4$

15 Talcott JB, Witton C, McLean MF, et al. Dynamic sensory sensitivity and children's word decoding skills. Proc Natl Acad Sci U S A 2000; 97(6):2952-2957

16 Zaidan E, Baran JA. Gaps-in-noise (GIN) test results in children with and without reading disabilities and phonological processing deficits. Int J Audiol 2013 Feb, 52(2):113-23

17 Chermak GD, Lee J. Comparison of children's performance on four test of temporal resolution. J Am Acad Audiol 2005 Sep; 16(8): 554-63

18 Murphy CFB, Schochat E. Correlations between reading, phonological awareness and auditory temporal processing. Pro Fono 2009;21(1):13-8

19 Samelli AG, Schochat E. The gaps-in-noise test: gap detection thresholds in normal-hearing young adults. Int J Audiol 2008; 47(5):238-245

20 Amaral MIR, Colella-Santos MF. Temporal resolution: performance of school-aged children in the GIN-gaps-in-noise test. Braz J Otorhinolaryngol 2010;76(6):745-752 